\title{
Mathematical Model and Non-Pharmaceutical Control of the Coronavirus 2019 Disease in Madagascar
}

\author{
Angelo Raherinirina, Fontaine Rafamatanantsoa, Tsilefa Stefana Fandresena, \\ Rivo Andry Rakotoarivelo
}

University of Fianarantsoa, Fianarantsoa, Madagascar

Email: angelo.raherinirina@gmail.com

How to cite this paper: Raherinirina, A., Rafamatanantsoa, F., Fandresena, T.S. and Rakotoarivelo, R.A. (2021) Mathematical Model and Non-Pharmaceutical Control of the Coronavirus 2019 Disease in Madagascar. Open Journal of Modelling and Simulation, 9, 259-274.

https://doi.org/10.4236/ojmsi.2021.93017

Received: June 4, 2021

Accepted: July 16, 2021

Published: July 19, 2021

Copyright () 2021 by author(s) and Scientific Research Publishing Inc. This work is licensed under the Creative Commons Attribution International License (CC BY 4.0).

http://creativecommons.org/licenses/by/4.0/

(c) (i) Open Access

\begin{abstract}
For Madagascar, with the uncertainty over vaccines against the novel coronavirus 2019 and its variants, non-pharmaceutical approach is widely used. Our objective is to propose a mathematical control model which will serve as a tool to help decision-makers in the strategy to be implemented to better face the pandemic. By separating asymptomatic cases which are often not reported and symptomatic who are hospitalized after tests; we develop a mathematical model of the propagation of covid-19 in Madagascar, by integrating control strategies. We study the stability of the model by expressing the basic reproduction number using the next-generation matrix. Simulation with different parameters shows the effects of non-pharmaceutical measures on the speed of the disease spread. By integrating a control parameter linked to compliance with barrier measures in the virus propagation equation, we were able to show the impacts of the implementation of social distancing measures on the basic reproduction number. The strict application of social distancing measures and total confinement is unfavorable for economic situation even if they allow the contamination to be reduced quickly. Without any restrictions, the disease spreads at high speed and the peak is reached fairly quickly. In this condition, hospitals are overwhelmed and the death rate increases rapidly. With $50 \%$ respect for non-pharmaceutical strategies such as rapid detection and isolation of positive cases and barrier gestures; the basic reproduction number $R_{0}$ can go down from 3 to 1.7. The pressures on the economic and social situation are rather viable. It is the most suitable for the Malagasy health system. The results proposed are a way to control the spread of the disease and limit its devastation in a country like Madagascar.
\end{abstract}

\section{Keywords}

Covid-19 Madagascar, Epidemic Model, Non-Pharmaceutical Control, 
Equilibrium Points, Stability Analysis, Reproduction Number

\section{Introduction}

The severe coronavirus 2019 (Covid-19) disease has now lasted for over a year. Since it was first reported in December 2019 in China, the virus has quickly spread to other parts of the world taking on pandemic proportions [1] [2]. Madagascar recorded its first case of coronavirus in March 2020 in an immigrant who visited the country. This new SARSCov- 2 coronavirus has been of great interest to scientists around the world because of its high contagiousness [3]. It is a respiratory virus that is spread mainly through contact with an infected person through respiratory droplets produced when a person coughs or sneezes or through droplets of saliva or nasal secretions. It is important that everyone observes good respiratory hygiene rules.

Hit with the second wave since April 2021, Madagascar experienced its highest rate of positivity and mortality in May 2021. Compared to data from the first wave, the virus becomes more contagious and deadly, see Figure 1 and Figure 2.

By multidisciplinary publications, several models have been proposed to study this phenomenon in several countries. In a neighboring island, Manou-Abi et al. proposed a modified SEIR model to analyze and predict the spread of covid-19 in the French overseas department of Mayotte [4]. These models are very interesting for understanding and forecasting the dynamics of the pandemic.

Generally, the modeling work consists of studying the dynamics of virus propagation and offers predictions on the impacts. An important part is also to study the effects of the various attenuation measurements by simulation [5] [6].
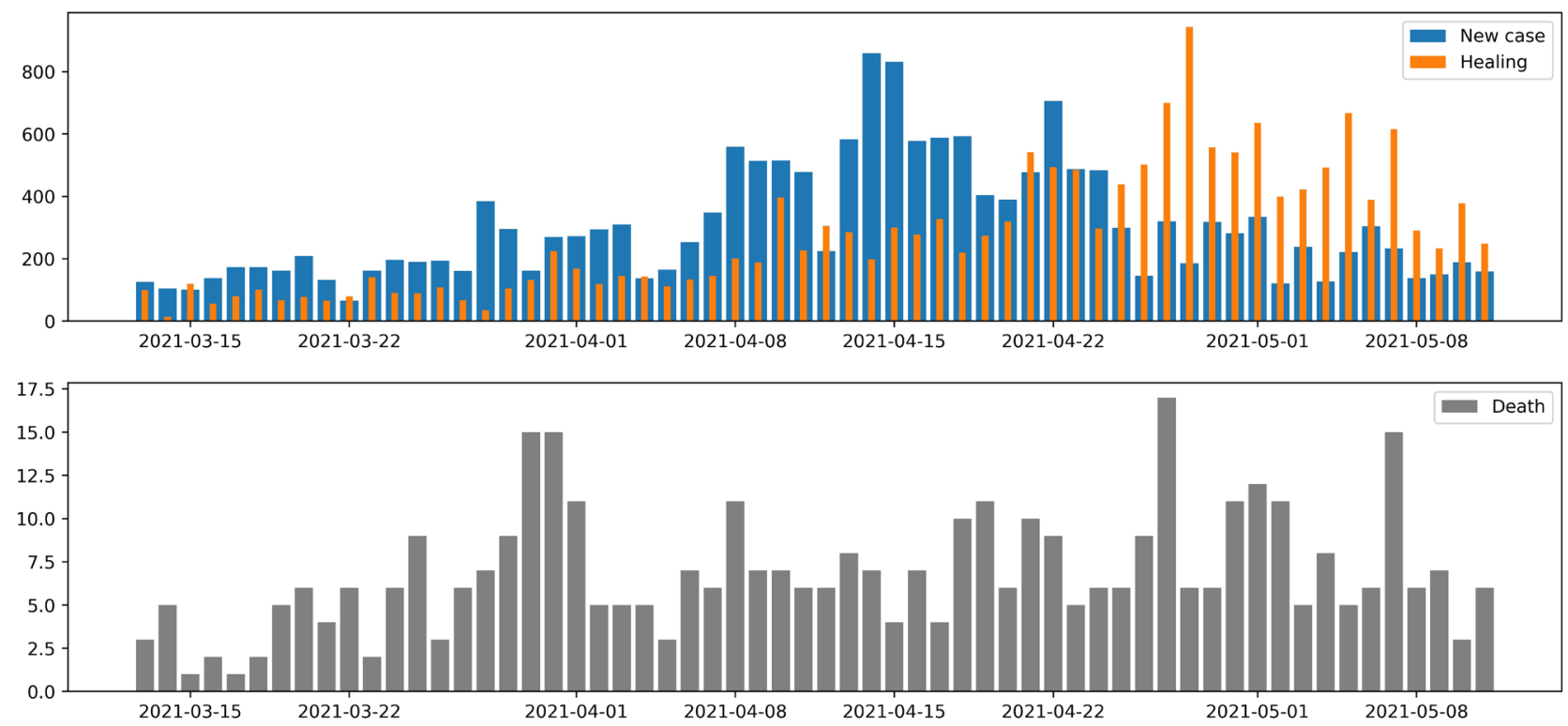

Figure 1. Weekly census of Covid-19 new cases in Madagascar from November 2020 until April 2021. Top: numbers of new cases and cured cases. Bottom: number of deaths. 

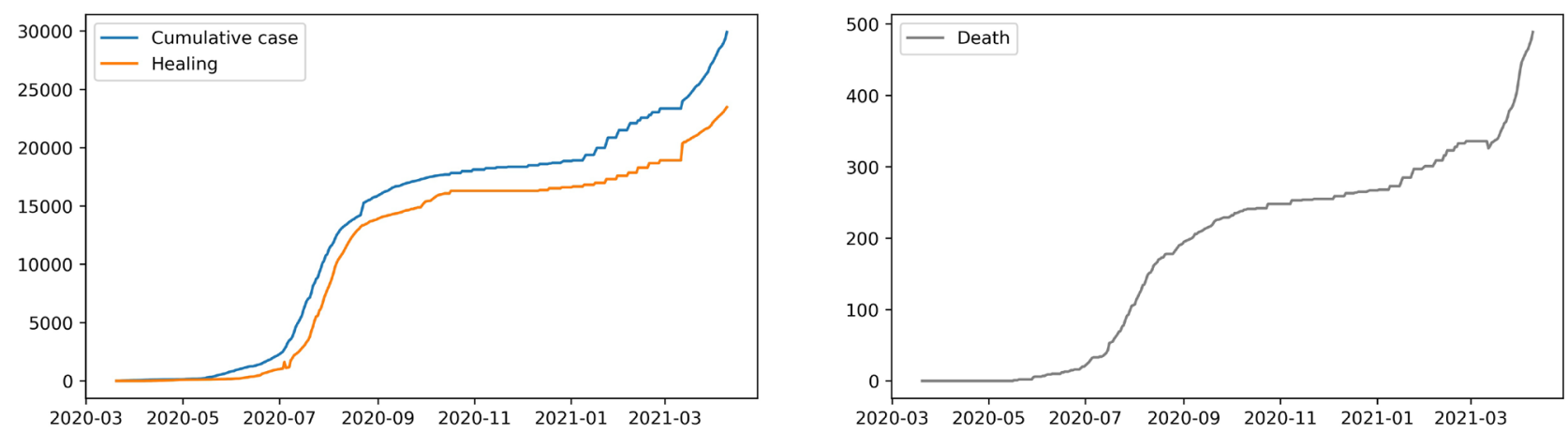

Figure 2. Cumulative cases observed from March 2020 to May 2021.

For Madagascar, in a recent work, we proposed a mathematical modeling framework for the propagation of Covid-19. Starting from a deterministic model, we have developed stochastic models which allow better representation of the context [7]. We inferred the models from official data released during the first wave of the epidemic in March 2020. For the proposed models, we estimated the basic reproduction number to assess the contagiousness of the virus. We were unable to address the issues related to the controls and management of the pandemic.

Along with the efforts to find reliable supports including vaccines to fight against the coronavirus [8] [9]; the impacts of so-called non-pharmaceutical strategies are not negligible [10] [11]. Among these non-pharmaceutical interventions, we distinguish mitigation by wearing masks and barrier gestures, isolation of confirmed cases and containment. In this paper, we are interested in the impacts of non-pharmaceutical approaches on the spread of the disease. Analyzing the effects of non-pharmaceutical measures constitutes a strategy to control the progression of the disease [12].

Mathematical models are powerful tools that have proven to be important in past epidemiological disasters such as malaria, measles or cholera. They contribute to the understanding of disease dynamics and provide useful predictions about the potential transmission of a disease that can provide valuable information to public health decision makers [13] [14] [15]. In addition to forecasting, mathematical models make it possible to study the different possible scenarios on the dynamics of the pandemic. These give us information on good ways to control the disease. In the case of Covid-19, some studies have been done on this subject [16] [17] [18].

To analyze its dynamics in Madagascar, we consider a mathematical model of the spread of the SARS-COV2 virus integrating the impacts of restrictive measures [19]. By taking the modified SEIR model that we developed in [7], we study the impacts of containment measures and the multiplication of tests on the values of the basic reproduction number. Similar studies have been done in a few African countries [20] [21]. Authors present models that take into account the response strategy of countries to the evolution of the disease. Our goal is to carry out the same study for Madagascar. 
After presenting the model with its hypotheses, we propose to fit the model with the data of Covid-19 in Madagascar. We calculate the expression of the basic reproduction number $R_{0}$ using the parameters of the model. Next, we simulate interventions with different parameter values and compare some repost strategies.

\section{The SEIR Modified Model of Covid-19 in Madagascar}

To describe the dynamics of Covid-19 in the context of Madagascar, we propose a seven compartment model, see Figure 3:

- $S$ (Susceptible): individuals who are not infected but at some risk of becoming infected;

- $E$ (Exposed): individuals who have been in contact with the virus but not yet infectious until after a latency period;

- $I$ (Infected): individuals who can infect and are not yet hospitalized or isolated;

- $H$ (Hospitalized): confirmed and hospitalized (isolated) cases;

- A (Asymptomatic): infected individuals but do not have symptoms (not isolated);

- $C$ (Cured): healed confirmed cases;

- $\quad D$ (Death): deaths due to Covid-19.

By making simplifying hypotheses on the transmission of the virus and the evolution of the disease on a Covid-19 patient [4]; the parameters of the model are summarized in Table 1. However, we would like to point out that the values of the parameters proposed are taken at the end of clinical observations in a few hospitals in Madagascar in collaboration with specialists.

At a time $t$, we denote $S(t), E(t), I(t), A(t), H(t), C(t)$ and $D(t)$ the number of individuals in the seven compartments. As in all epidemiological compartmental models, we assume that the total number of population $N$ is constant and:

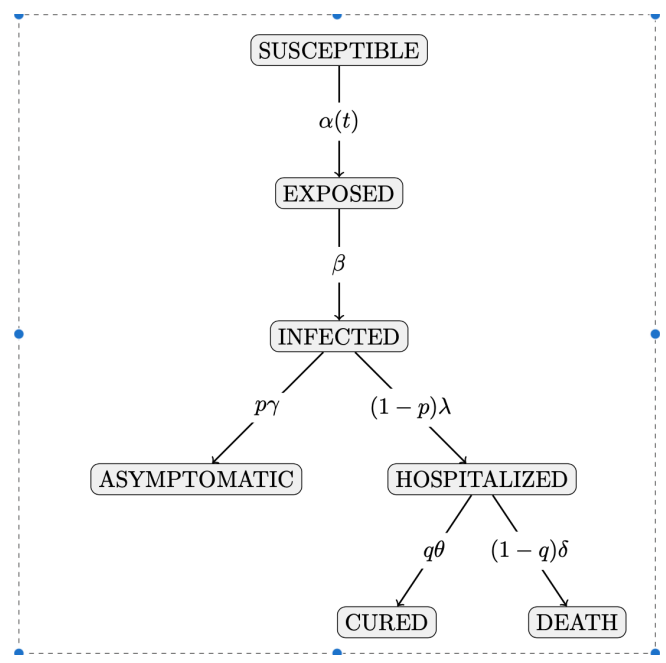

Figure 3. Modified SEIR model with seven compartments. 
Table 1. Parameters of the Covid-19 SEIR modified model.

\begin{tabular}{ccc}
\hline notation & description & range \\
\hline$\alpha$ & rate of spread of the epidemic & - \\
$p$ & proportion of asymptomatic cases & $35 \%-40 \%$ \\
$\beta$ & occurrence of symptoms & $4-5$ days \\
$\gamma$ & healing time for simple cases (not hospitalized) & $7-14$ days \\
$\theta$ & time to detect infectious diseases & $5-10$ days \\
$\delta$ & time for hospitalized cures & $20-40$ days \\
$q$ & time of infections causing death & $14-50$ days \\
\hline
\end{tabular}

$$
N=N(t)=S(t)+E(t)+I(t)+A(t)+H(t)+C(t)+D(t),
$$

for all $t$. In a small time interval $[t, t+\mathrm{d} t]$ the evolution of the system is described by the Equation (1):

$$
\left\{\begin{array}{l}
\frac{\mathrm{d} S(t)}{\mathrm{d} t}=-\alpha \frac{S(t) I(t)}{N}, \\
\frac{\mathrm{d} E(t)}{\mathrm{d} t}=\alpha \frac{S(t) I(t)}{N}-\beta E(t), \\
\frac{\mathrm{d} I(t)}{\mathrm{d} t}=\beta E(t)-p \gamma I(t)-(1-p) \lambda I(t), \\
\frac{\mathrm{d} A(t)}{\mathrm{d} t}=p \gamma I(t), \\
\frac{\mathrm{d} H(t)}{\mathrm{d} t}=(1-p) \lambda I(t)-(1-q) \delta H(t)-q \theta H(t), \\
\frac{\mathrm{d} C(t)}{\mathrm{d} t}=q \theta H(t), \\
\frac{\mathrm{d} D(t)}{\mathrm{d} t}=(1-q) \delta H(t) .
\end{array}\right.
$$

with $(1-p) \lambda I(t) \mathrm{d} t$ the number of confirmed cases (isolated, hospitalized), $p \gamma I(t) \mathrm{d} t$ the number of asymptomatic cases and $\alpha(S(t) I(t)) / N \mathrm{~d} t$ the number of new infections in the interval $[t, t+\mathrm{d} t]$.

The problem is well posed and the solutions exist given that the initial data are non-negative.

Let

$$
\Gamma=\left\{(S, E, I, A, H, C, D) \in \mathbb{R}_{7}^{+}: S^{*}+E^{*}+I^{*}+A^{*}+H^{*}+C^{*}+D^{*}=1\right\}
$$

with $S^{*}=S / N, \quad E^{*}=E / N, I^{*}=I / N, A^{*}=A / N, H^{*}=H / N, C^{*}=C / N$ and $D^{*}=D / N$ positively invariant and attractive in $\mathbb{R}_{7}^{+}$.

It is therefore sufficient to study the dynamics of the Equation (1), to understand the evolution of the pandemic.

The points of equilibrium corresponding are obtained by 


$$
\mathrm{d} S / \mathrm{d} t=\mathrm{d} E / \mathrm{d} t=\mathrm{d} I / \mathrm{d} t=\mathrm{d} A / \mathrm{d} t=\mathrm{d} H / \mathrm{d} t=\mathrm{d} C / \mathrm{d} t=\mathrm{d} D / \mathrm{d} t=0 .
$$

The equilibrium point $E_{0}$ is a solution in the stationary state

$$
E_{0}=\left(S_{0}, E_{0}, I_{0}\right)=\left\{S_{0} \leq N, 0,0\right\} .
$$

At this point there is no epidemic; nothing is happening in the population.

\section{Model Analysis}

The basic reproduction number $R_{0}$ corresponding to the model is estimated using officially recorded data. It is defined as the average number of secondary cases produced by a fundamental case over its infectious period. It therefore represents the initial growth rate of the epidemic. According to this model, the estimated value of $R_{0}$ during the first wave in 2020 is 1.2 [7]. For this second wave, the temporal estimate of $R_{0}$ is described by Figure 4 .

Another technique developed by Driessche et al. consists of expressing $R_{0}$ using the next-generation matrix by following the algorithm [22]:

1) Identify infected compartments,

2) Identify terms that correspond to new infections and place them in a matrix $F$, and identify terms that correspond to the transfer of existing infections and place them in a matrix $V$;

3) Calculate the disease-free equilibrium;

4) Calculate the Jacobian matrix of $\mathrm{F}$ (respectively of $V$ ) at disease-free equilibrium;

5) Invert $V$ to obtain $V^{-1}$;

6) Calculate $\mathrm{FV}^{-1}$;

7) $R_{0}$ is the spectral radius of the next-generation matrix $F^{-1}$.

The system (1) is made up of three infected states $E, I, H$ and four uninfected states $S, A, C, D$.

Consider the linearized infection subsystem:

$$
\left\{\begin{array}{l}
E=\alpha I-\beta E \\
I=\beta E-p \gamma I-(1-p) \lambda I \\
H=(1-p) \lambda I-(1-q) \delta H-q \theta H
\end{array}\right.
$$

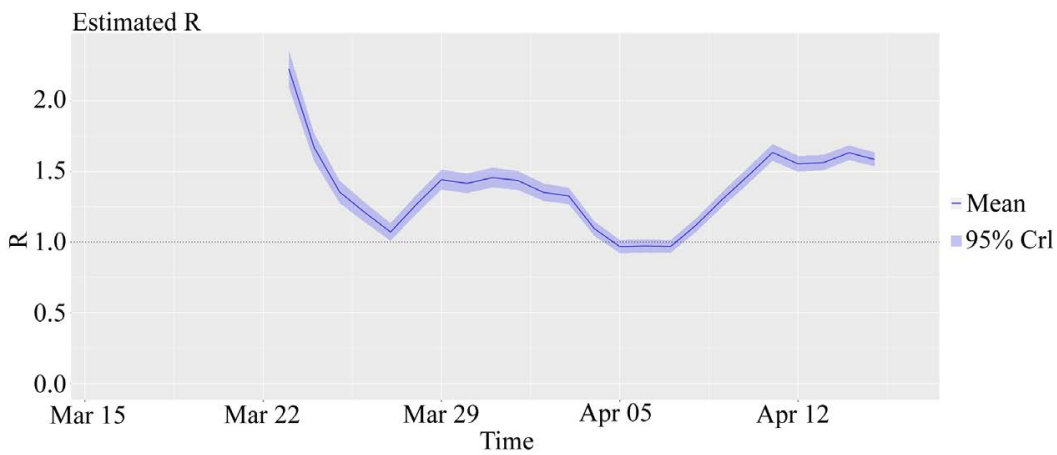

Figure 4. Temporal estimation of $R_{0}$ during the second wave. On average, $R_{0}$ is estimated at 1.45 with a confidence interval of $95 \%$. 
By denoting by $x$ the vector formed by the numbers of individuals in each compartment; decompose the linearized subsystem by

$$
\dot{x}=(F-V) x,
$$

where the matrix $F$ corresponds to transmissions and $V$ to transitions.

Thus, all epidemiological events that lead to new infections are incorporated into the model by $F$, and all other events by $V$ with

$$
F=\left(\begin{array}{ccc}
0 & \alpha & 0 \\
0 & 0 & 0 \\
0 & 0 & 0
\end{array}\right) \text { and } V=\left(\begin{array}{ccc}
-\beta & 0 & 0 \\
\beta & p \gamma+(1-p) \lambda & 0 \\
0 & -(1-p) \lambda & q \theta+(1-q) \delta
\end{array}\right)
$$

Thus, the sixth step of the above algorithm is written

$$
F V^{-1}=\left(\begin{array}{ccc}
\frac{\alpha}{p \gamma+(1-p) \lambda} & \frac{\alpha}{p \gamma+(1-p) \lambda} & 0 \\
0 & 0 & 0 \\
0 & 0 & 0
\end{array}\right) .
$$

The basic reproduction number $R_{0}$ is the spectral radius of the next-generation matrix $F V^{-1}: \rho\left(F V^{-1}\left(E_{0}\right)\right)$.

We find

$$
R_{0}=\frac{\alpha}{p \gamma+(1-p) \lambda}
$$

The expression of $R_{0}$ in Equation (4) suggests a way to control it. Indeed, higher values of the transmission rate $\alpha$ and a smaller values of the proportion $p$ of isolated cases and the delay of the isolation allow acceleration of the epidemic.

On the other hand, with a low value of $\alpha$ and a decision to quickly isolate the infectious cases, the epidemic disappears.

- If $\alpha<p \gamma+(1-p) \lambda$, i.e. $R_{0}<1$, the disease-free equilibrium is the only equilibrium and the diseases disappear;

- If $\alpha>p \gamma+(1-p) \lambda$, i.e. $R_{0}>1$, the epidemic persists in the population.

The only way to stop the transmission of the epidemic without limiting the dynamics of the populations is the rapid isolation of a sufficient proportion of infectious individuals.

The nature of the stability of the disease-free equilibrium (DFE) is determined by the eigenvalues of the Jacobian matrix

$$
J=\left(\begin{array}{lll}
-\partial f_{1} / \partial S & -\partial f_{1} / \partial E & -\partial f_{1} / \partial I \\
-\partial f_{2} / \partial S & -\partial f_{2} / \partial E & -\partial f_{2} / \partial I \\
-\partial f_{3} / \partial S & -\partial f_{3} / \partial E & -\partial f_{3} / \partial I
\end{array}\right)
$$

where

$$
f_{1}=\mathrm{d} S / \mathrm{d} t, \quad f_{2}=\mathrm{d} E / \mathrm{d} t \text { and } f_{3}=\mathrm{d} I / \mathrm{d} t .
$$

We have 


$$
J=\left(\begin{array}{ccc}
-\alpha I / N & 0 & -\alpha S / N \\
\alpha I / N & -\beta & \alpha S / N \\
0 & \beta & -p \gamma-(1-p) \lambda
\end{array}\right) .
$$

Considering $E_{0}$, the DFE point $E_{0}$ is asymptotically stable if the real parts of the eigenvalues are all negative and unstable if the real parts are positive [23] [24]. Results show that the stable case corresponds to $R_{0}<1 \quad$ [14].

\section{Herd Immunity and Consideration of Social Distancing Measures}

Various studies have shown that the basic reproduction number is proportional to the proportion of individuals susceptible to infection [25]. The lower the proportion of the population susceptible to infection, the lower the risk of emergence.

For a value $R_{0}\left(t_{0}\right)$, we can calculate the proportion $v$ that must be immunized to prevent the epidemic, i.e. vary $R_{0}$ below a threshold of 1:

$$
R_{0}\left(t_{0}\right)(1-v)<1 \text {. }
$$

We obtain:

$$
v>1-\frac{1}{R_{0}\left(t_{0}\right)}
$$

The value of $v$ is the threshold value for group immunity to be high enough to prevent the spread of the virus. This strategy coincides with the policy of mass vaccination.

According to the work of Kermarck et al. [25], the proportion of the population that will have been infected in the end, denote by $q^{*}$ satisfies the equation below:

$$
R_{0} q^{*}+\log \left(1-q^{*}\right)=0
$$

Figure 5 proposes a numerical resolution of Equations (5) and (4). We note that the proportion of affected individuals is restricted as the number of immunized

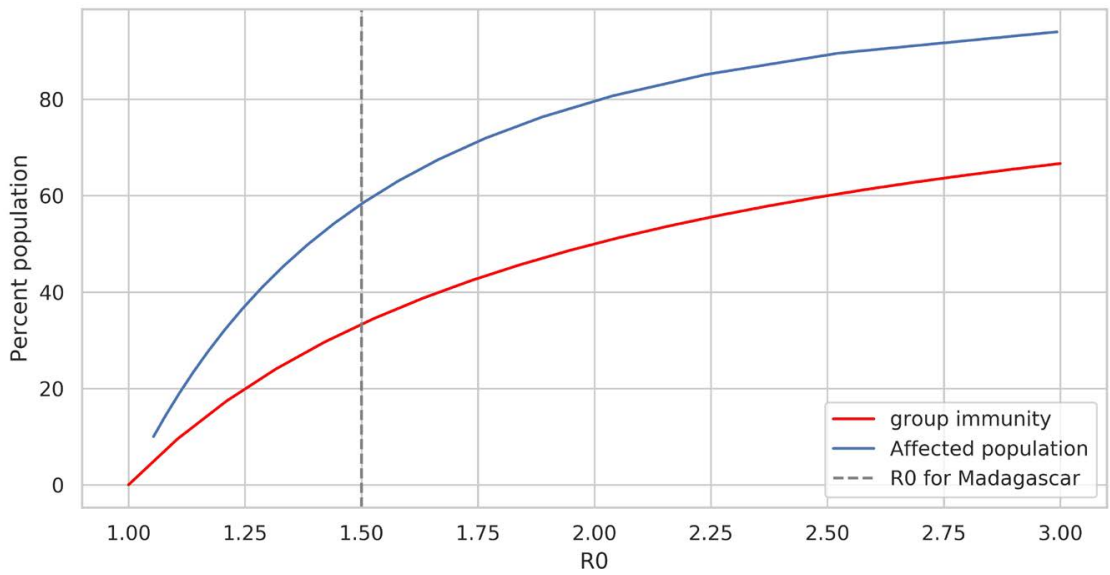

Figure 5. Herd immunity and proportion of infected populations. 
individuals increases. In practice, this makes it possible to know the number of individuals to be vaccinated in order to prevent the spread of the disease.

For the case of the coronavirus in Madagascar, with the parameter $R_{0}=1.5$ calculated with data from the second wave; it is estimated that an infection rate of around $60 \%$ must be achieved to ensure extinction of the disease. This corresponds to vaccinating at least $35 \%$ of the population, see Figure 5 .

In the following, we will integrate the effects of social distancing measures in our model of the spread of the disease. With the help of a simulation, we prove that the strict respect of the barrier gestures largely influences the basic reproduction rate $R_{0}$, see Figures 6-8.

Our ideas are based on the assumption that the Covid-19 transmission rate $\alpha(t)$ at time $t$ is constant until the time $t^{*}$ when the control measures are introduced. Afterwards, it decreases exponentially according to the intensity of compliance with the distancing measures [4]. It is assumed that a proportion $c \in[0,1)$ of new infections is reduced over a period of time $T=t-t^{*}$ where $t^{*}$ is the moment when the control measures are introduced:

$$
\alpha(t)=(1-c) \alpha, \forall t \in\left[t^{*}, t^{*}+T\right] .
$$

Assuming that the restrictive measures last for a period $n$, we can write

$$
\alpha(t)=\alpha(1-c)^{n}=\alpha \mathrm{e}^{\ln (1-c)^{n}}=\alpha \mathrm{e}^{n q}, n \geq 1
$$
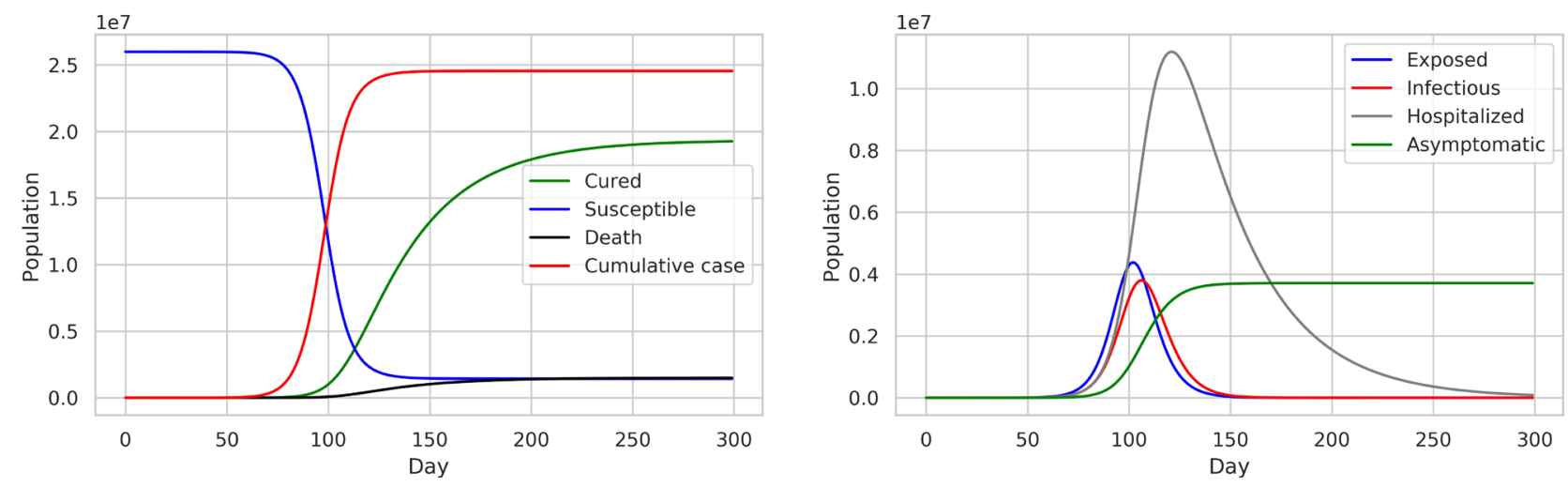

Figure 6. Spread of the disease without applying barrier gestures $(c=0)$.
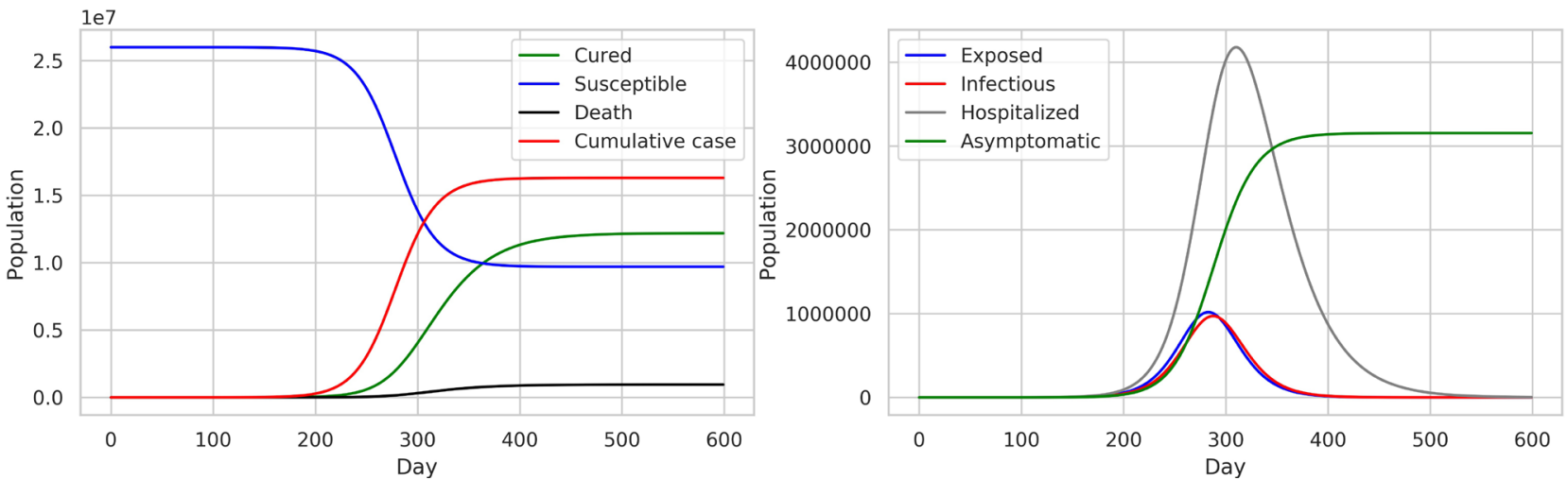

Figure 7. Spread of the virus with respect for barrier and containment measures, at $50 \%(c=0.5)$. 

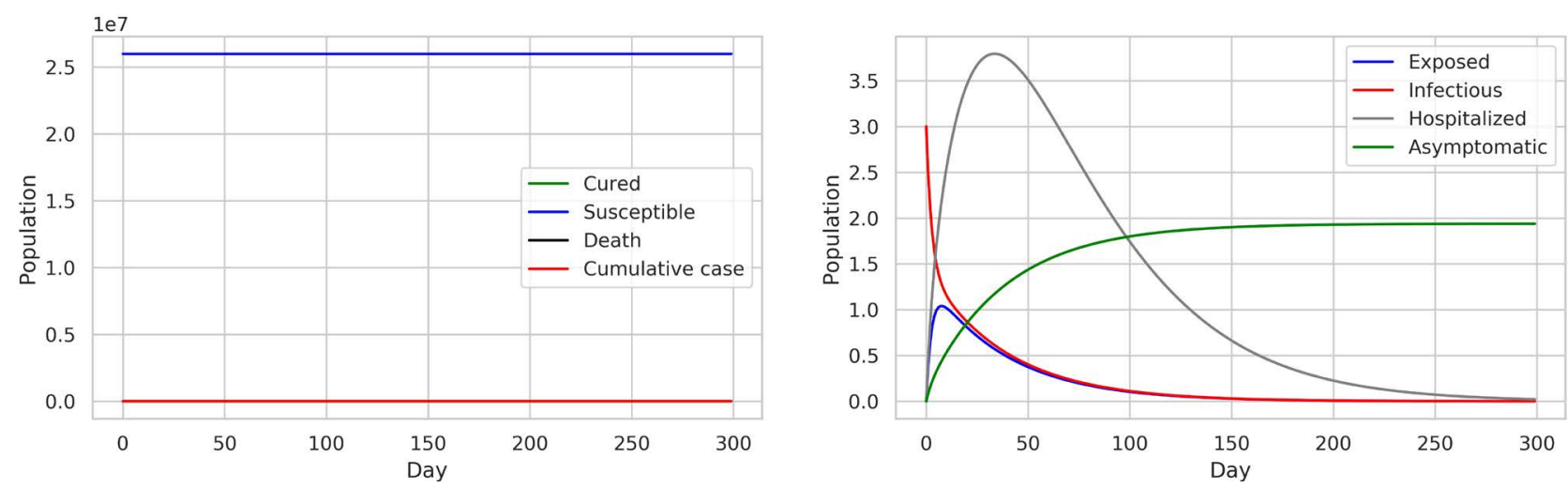

Figure 8. Spread of the virus with respect for barrier and containment measures, at $75 \backslash \%(c=0.75)$.

where

$$
q=\ln (1-c) \in[0,1)
$$

is a attenuation control parameter.

Thus, the model is controlled by the parameter $c(6)$ by integrating

$$
\alpha(t)=\alpha \mathrm{e}^{n q}, n \leq 0, \forall t \in\left[t^{*}, t^{*}+T\right]
$$

into the disease propagation Equation (1).

We have run simulations with some reference values of the control parameter

$c$. The results are described below.

- Figure 6: By taking $c=0$, i.e. without restrictive measures or respect for barrier gestures, we estimated $R_{0}=3$. The disease spreads quickly and the peak is reached within a short time; 25 days on average. In this condition, the health system will be quickly overwhelmed. The pandemic causes much more damage.

- Figure 7: for $c=0.50$. Strict compliance with barrier gestures is $50 \%$. The estimated basic reproduction number is $R_{0}=1.53$. The peak was reached more slowly in 75 days on average. Thus, the health system could prepare itself gradually in order to avoid the catastrophe. With a little freedom, the pressure of restrictions on the economy is more or less acceptable. This corresponds to the situation in Madagascar during the first wave.

- Figure 8: for $c=0.75$. Strict compliance with barrier gestures is $75 \%$. The effects on the management of the pandemic are positive but the economic and social impacts are intense.

\section{Positive Cases Detection Strategy and Isolation}

Another measure to stop the spread of the virus is to detect positive cases as early as possible [26]. Various studies in several countries show that isolation is one of the fastest ways to prevent the spread of Covid-19 [27] [28]. This strategy is effective but very expensive, given the cost of the tests.

By controlling the parameter $\lambda$ of Equation (4), we were able to highlight the impact of the rapid detection of positive cases and isolation in the dynamics of 
virus propagation. Simulation show that: from an epidemic situation with $R_{0}=3$; with application of barrier gestures at $50 \%$; if we can quickly detect and isolate positive cases; we can reduce the base reproduction number down to 1.71 after a few days, see Figure 9.

In the same condition on the application of social distancing measures, if we manage to quickly detect the $80 \%$ of positive cases, we can reduce $R_{0}$ down to 0.78; see Figure 10. It is therefore confirmed that the rapid detection of positive cases is an effective way to limit the propagation of the virus and the mortality rate due to Covid-19.

Considering the high cost of the tests, the implementation of this technique seems impossible for underdeveloped countries like Madagascar. Studies have been done for clinical signs that can help specialists in anticipation and decision making [26].

Thus, any non-pharmaceutical control strategy for the Covid-19 pandemic consists in reducing the basic reproduction number $R_{0}$ below the threshold value 1 by respecting social distancing measures [29]. Several countries have resorted to a total containment measure to quickly flex the epidemiological curve. Certainly, these measures are very expensive but its application requires a minimum of time to have the best results [30]. Otherwise, once the restrictions are lifted, the epidemic starts to rise sharply again, see Figure 11 . This only worsens the health and economic situation of the population.
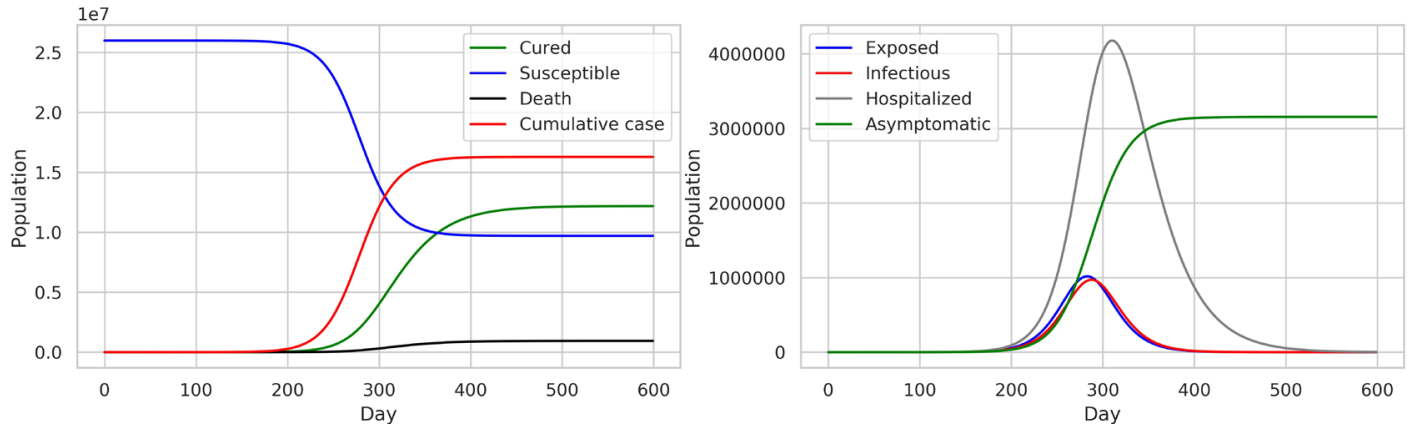

Figure 9. Reduction of the basic reproduction number $R_{0}$ from 3 to 1.71 by the rapid detection and isolation of $50 \%$ of positive cases and $50 \%$ compliance with social distancing measures.
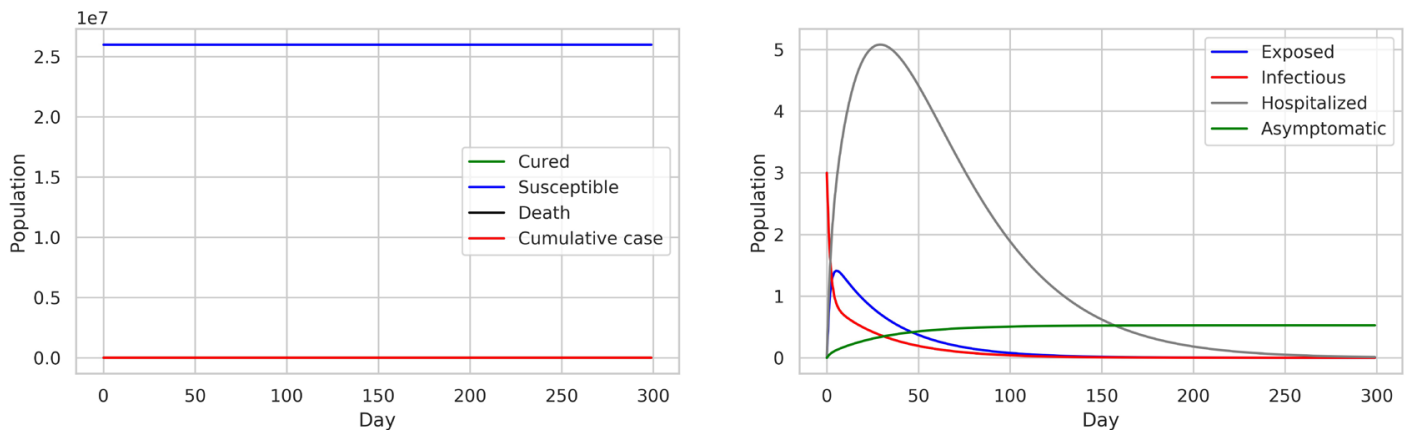

Figure 10. Reduction of the basic reproduction number $R_{0}$ from 3 to 0.78 by the rapid detection and isolation of $80 \%$ of positive cases and $50 \%$ compliance with social distancing measures. 

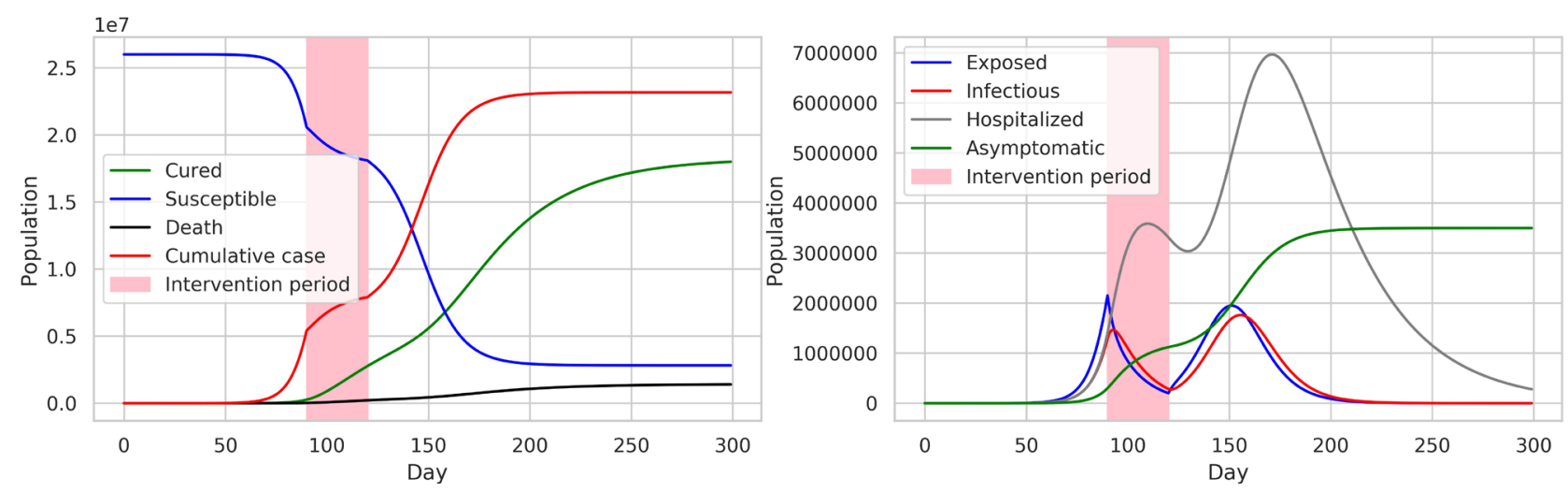

Figure 11. Starting from $R_{0}=3$, simulation of the model with compliance with the restriction measures at $80 \%$ for a short period of 30 days.

The imposition of restrictive measures related to pandemic Covid-19 requires flexibility with regard to these economic and social impacts. In a poor country like Madagascar, too much restriction only makes the situation worse [31]. Many people have to go out to work to get something to eat. Decision-makers must be flexible and consider several parameters other than epidemiological parameters. This work is a helpful tool.

\section{Conclusions}

The coronavirus 2019 with all its variants is surely the most serious and the most worrying of the epidemics that have existed since our days. He has already killed several thousand people all over the world. Several countries are more affected than others because of failures in their health systems. Always in search of miracle cures as well as effective vaccines, the recourse to the non-pharmaceutical approach is the most effective to limit the human damage of the disease. Often costly and difficult to gain acceptance in practice, the containment measure has enabled several countries to slow the spread of the virus while implementing a policy of mass vaccination and by population segment.

In addition to modeling and forecasting, the objective of this paper is to provide tools to help decision-makers in managing the health crisis. Many people still hesitate between the effectiveness and the material and moral costs of the restrictive measures imposed to reduce the contagiousness of the virus. Our results show that the effective implementation of barrier gestures and social distancing largely influence the dynamics of the spread of the coronavirus.

The proposed model is composed of seven compartments in accordance with the approach to managing the coronavirus in Madagascar. By inferring the model with epidemiological data from Madagascar during this second wave, we have estimated the basic reproduction number: $R_{0}=1.45$. Compared to the estimated value with data from the first wave, this value is higher. This confirms that this second wave of the pandemic is more contagious, more deadly than the first wave in 2020 . 
To assess the impact of the application of barrier gestures, we integrate a control parameter in the expression of the rate of transmission of the coronavirus disease model. This parameter measures with a proportion the intensity of compliance with restrictive measures.

Simulations with some reference values show that the basic reproduction number gradually decreases depending on the strict application of social distancing measures. In fact, compliance at $50 \%$ of the restriction measures can reduce the basic reproduction number from 3 to 1.7 .

The rapid detection of positive cases through massive testing is also an effective strategy in the management of a pandemic such as Covid-19.

Unfortunately this strategy is very expensive and difficult to implement, especially in poor countries. However, using a simulation, we have shown that the application of this technique for Madagascar can radically eliminate the epidemic.

Thus, with the technique which consists in expressing the basic reproduction number by the next-generation matrix, we were able to integrate control parameters on the propagation of Covid-19 in Madagascar. Coupled with the parameter for monitoring compliance with barrier measures, the parameter on the detection rate of positive cases are the two key parameters for controlling the spread of the coronavirus according to our model. This is part of the contributions of this paper on the analysis and modeling of the dynamics of Covid-19 in Madagascar.

Our control model focuses on the intensity of the application of barrier measures which are often very costly socially and economically. This is paradoxical because the application of these measures must last a certain time, at least 3 months to be more effective. The hasty removal of restrictions allows an exponential increase in contamination.

\section{Conflicts of Interest}

The authors declare no conflicts of interest regarding the publication of this paper.

\section{References}

[1] Sadeghi, D., Taati, M. and Satarzadeh, N. (2020) COVID-19 (Coronavirus Disease 2019): A New Coronavirus Disease. Infection and Drug Resistance, 13, 2819-2828. https://doi.org/10.2147/IDR.S259279

[2] World Health Organisation (2021) Coronavirus Disease (COVID-19) Weekly Epidemiological Update and Weekly Operational Update.

https://www.who.int/emergencies/diseases/novel-coronavirus-2019/situation-report $\underline{s}$

[3] Mohapatra, R., Pintilie, L., Kandi, V., Sarangi, A., Das, D., Sahu, R. and Lina, P. (2020) The Recent Challenges of Highly Contagious COVID-19, Causing Respiratory Infections: Symptoms, Diagnosis, Transmission, Possible Vaccines, Animal Models, and Immunotherapy. Chemical Biology \& Drug Design, 96, 1187-1208. https://doi.org/10.1111/cbdd.13761 
[4] Manou-Abi, S. and Balicchi, J. (2020) Analysis of the COVID-19 Epidemic in French Overseas Department Mayotte Based on a Modified Deterministic and Stochastic SEIR Model. https://doi.org/10.1101/2020.04.15.20062752

[5] Kyrychko, Y., Blyuss, K. and Brovchenko, I. (2020) Mathematical Modelling of the Dynamics and Containment of COVID-19 in Ukraine. Scientific Reports, 10, Article No. 19662. https://doi.org/10.1038/s41598-020-76710-1

[6] Ndairou, F., Area, I., Nieto, J. and Torres, D. (2020) Mathematical Modeling of COVID-19 Transmission Dynamics with a Case Study of Wuhan. Chaos, Solitons \& Fractals, 135, Article ID: 109846. https://doi.org/10.1016/j.chaos.2020.109846

[7] Raherinirina, A., Fandresena, T., Hajalalaina, A., Ra-betafika, H., Rakotoarivelo, R. and Rafamatanantsoa, F. (2021) Probabilistic Modelling of COVID-19 Dynamic in the Context of Madagascar. Open Journal of Modelling and Simulation, 9, 211-230. https://doi.org/10.4236/ojmsi.2021.93014

[8] Sharma, O., Sultan, A., Ding, H. and Triggle, C. (2020) Review of the Progress and Challenges of Developing a Vaccine for COVID-19. Frontiers in Immunology, 11, Article ID: 585354. https://doi.org/10.3389/fimmu.2020.585354

[9] Safar, H., Mustafa, A. and McHugh, T. (2020) COVID-19 Vaccine Development: What Lessons Can We Learn from TB? Annals of Clinical Microbiology and Antimicrobials, 19, Article No. 56. https://doi.org/10.1186/s12941-020-00402-x

[10] Imai, N., Gaythorpe, K., Abbott, S., Bhatia, S., Van Elsland, S., Prem, K., Liu, Y. and Ferguson, N. (2020) Adoption and Impact of Non-Pharmaceutical Interventions for COVID-19. Wellcome Open Research, 5, 59. https://doi.org/10.12688/wellcomeopenres.15808.1

[11] Lai, S., Ruktanonchai, N., Zhou, L., Prosper, O., Luo, W., Floyd, J., Wesolowski, A., Santillana, M., Zhang, C., Du, X., Yu, H. and Tatem, A. (2020) Effect of Non-Pharmaceutical Interventions to Contain COVID-19 in China. Nature, 585, 410-413. https://doi.org/10.1038/s41586-020-2293-x

[12] European Centre for Disease Prevention and Control (2020) Guidelines for Non-Pharmaceutical Interventions to Reduce the Impact of COVID-19 in the EU/EEA and the UK.

https://www.ecdc.europa.eu/en/publications-data/covid-19-guidelines-non-pharma ceutical-interventions

[13] Nyarko, C., Nyarko, P., Ampofi, I. and Asante, E. (2020) Modelling Transmission of Buruli Ulcer in the Central Region of Ghana. Mathematical Modelling and Applications, 5, 221-230.

[14] Mitku, S. (2017) Mathematical Modeling and Simulation Study for the Control and Transmission Dynamics of Measles. American Journal of Applied Mathematics, 5, 99-107. https://doi.org/10.11648/j.ajam.20170504.11

[15] Fekadu, T. (2020) Mathematical Model of Controlling the Spread of Malaria Disease Using Intervention Strategies. Pure and Applied Mathematics, 9, 101-108. https://doi.org/10.11648/j.pamj.20200906.11

[16] Rotich, K., Lagat, R. and Choge, P. (2020) Mathematical Modeling of Covid-19 Disease Dynamics and Analysis of Intervention Strategies. Mathematical Modelling and Applications, 5, 176-182. https://doi.org/10.11648/j.mma.20200503.16

[17] Zamir, M., Shah, Z., Nadeem, F., Memood, A., Alrabaiah, H. and Kumam, P. (2020) Non Pharmaceutical Interventions for Optimal Control of COVID-19. Computer Methods and Programs in Biomedicine, 196, Article ID: 105642. https://doi.org/10.1016/j.cmpb.2020.105642 
[18] Andrew, M., Jesse, S., Saunak, S., Fridtjof, T. and Juliette, H.T. (2021) Estimating the Effect of Social Distancing Interventions on COVID-19 in the United States. American Journal of Epidemiology, 1.

[19] Calistus, N., Enahoro, I., Steffen, E., Matthew, S., Chandini, R., Matthew, H. and Abba, B. (2020) Mathematical Assessment of the Impact of Non-Pharmaceutical Interventions on Curtailing the 2019 Novel Coronavirus. Mathematical Biosciences, 325, Article ID: 108364. https://doi.org/10.1016/j.mbs.2020.108364

[20] Abdoul, W., Badjo, K., Moustapha, D., Rabi, H. and Bisso, S. (2020) Contribution to the Mathematical Modeling of COVID-19 in Niger. Applied Mathematics, 11, 427-435. https://doi.org/10.4236/am.2020.116030

[21] Eric, N., Ernest, D., Daniel and Ekow, B. (2020) Modelling the Dynamics of COVID-19 Disease with Contact Tracing and Isolation in Ghana. Mathematical Modelling and Applications, 5, 146-155.

https://doi.org/10.11648/j.mma.20200503.13

[22] van den Driessche, P. and Watmough, J. (2002) Reproduction Numbers and Sub-Threshold Endemic Equilibria for Compartmental Models of Disease Transmission. Mathematical Biosciences, 180, 29-48. https://doi.org/10.1016/S0025-5564(02)00108-6

[23] Michel, A., Hou, L. and Liu, D. (2008) Fundamental Theory: The Principal Stability and Boundedness Results on Metric Spaces, In: Michel, A., Hou, L. and Liu, D., Eds., Stability of Dynamical Systems. Continuous, Discontinuous, and Discrete Systems (Systems \& Control: Foundations \& Applications), Birkhauser Boston, 71-148.

[24] Leontine, N., Timothee, M. and Sakamoto, N. (2019) Stability and Optimal Control of Tuberculosis Spread with an Imperfect Vaccine in the Case of Co-Infection with HIV. Open Journal of Modelling and Simulation, 7, 97-114.

https://doi.org/10.4236/ojmsi.2019.72005

[25] Kermack, W. and McKendrick, A. (1927) Contribution to the Mathematical Theory of Epidemic. Proceedings of the Royal Society of London, 115, 700-721.

https://doi.org/10.1098/rspa.1927.0118

[26] Raberahona, M., Rakotomalala, R., Rakotomijoro, E., Rahaingoalidera, T., Christophe E., Mamilaza, N., Razafindrabekoto, L., Rafanomezantsoa, E., Andriananja, V., Andrianasolo, R., Razafimahefa, H., Rakotoarivelo, R. and Randria, M. (2021) Clinical and Epidemiological Features Discriminating Confirmed COVID-19 Patients from SARS-CoV-2 Negative Patients at Screening Centres in Madagascar. International Journal of Infectious Diseases, 103, 6-8.

https://doi.org/10.1016/j.ijid.2020.11.151

[27] Molalegn, A., Tsige, H. and Kassahun, G. (2020) The Impact of Infective Immigrants and Self Isolation on the Dynamics and Spread of Covid-19 Pandemic: A Mathematical Modeling Study. Pure and Applied Mathematics Journal, 9, 109-117. https://doi.org/10.11648/j.pamj.20200906.12

[28] Wu, K.H., Hornsby, W., Klunder, B., Krause, A., Driscoll, A., Kulka, J., Bickett-Hickok, R., Fellows, A., Graham, S., Kaleba, E., Hayek, S., Shi, X., Sutton, N., Douville, N., Mukherjee, B., Jamerson, K., Brummett, C. and Willer, C. (2021) Exposure and Risk Factors for COVID-19 and the Impact of Staying Home on Michigan Residents. PLoS ONE, 16, e0246447.

https://doi.org/10.1371/journal.pone.0246447

[29] Chen, D. and Zhou, T. (2021) Evaluating the Effect of Chinese Control Measures on COVID-19 via Temporal Reproduction Number Estimation. PLoS ONE, 16, e0246715. https://doi.org/10.1371/journal.pone.0246715 
[30] Enahoro, A., Oluwaseun, S., Calistus, N. and Abba, B. (2020) Mathematical Modeling and Analysis of COVID-19 Pandemic in Nigeria. Mathematical Biosciences and Engineering, 17, 7192-7220.

[31] Teachout, M. and Zipfel, C. (2020) The Economic Impact of COVID-19 Lockdowns in Sub-Saharan Africa. International Growth Centre, London.

https://www.theigc.org/wp-content/uploads/2020/05/Teachout-and-Zipfel-2020-pol icy-brief-.pdf 